\title{
Early antibiotics administration during targeted temperature management after out-of-hospital cardiac arrest: a nationwide database study
}

Takashi Tagami ${ }^{1,2^{*}}$, Hiroki Matsui ${ }^{1}$, Masamune Kuno, Yuuta Moroe ${ }^{2}$, Junya Kaneko², Kyoko Unemoto², Kiyohide Fushimi ${ }^{3}$ and Hideo Yasunaga ${ }^{1}$

\begin{abstract}
Background: Patients resuscitated after cardiac arrest are reportedly at high risk for infection and sepsis, especially those treated with targeted temperature management (TTM). There is, however, limited evidence suggesting that early antibiotic use improves patient outcomes. We examined the hypothesis that early treatment with antibiotics reduces mortality in patients with cardiac arrest receiving $T$ TM.
\end{abstract}

Methods: We identified 2803 patients with cardiogenic out-of-hospital cardiac arrest (OHCA) that were treated with TTM and were admitted to 371 hospitals that contribute to the Japanese Diagnosis Procedure Combination inpatient database between July 2007 and March 2013. Of these, 1272 received antibiotics within the first 2 days (antibiotics) and 1531 did not (control). We generated 802 propensity score-matched pairs.

Results: There was no significant difference in 30-day mortality between the groups (control vs. antibiotics; $33.0 \%$ vs. $29.9 \%$; difference, $3.1 \%$; $95 \%$ confidence interval [CI], -1.4 to $7.7 \%, p=0.18$ ). Analysis using the hospital antibiotics prescribing rate as an instrumental variable showed that antibiotic use was not significantly associated with a reduction in 30-day mortality $(6.6 \%, \mathrm{Cl} 95 \%,-0.5$ to $13.7 \%, p=0.28)$. A subgroup analysis of patients who required extracorporeal membrane oxygenation (ECMO) indicated a significant difference in 30-day mortality between the 2 groups ( $62.9 \%$ vs. $43.5 \%$; difference $19.3 \%$, Cl $95 \%, 5.9$ to $32.7 \%, p=0.005$ ). In the instrumental variable model, the estimated reduction in 30-day mortality associated with antibiotics was $18.2 \%$ (Cl $95 \%$, 21.3 to $34.4 \%, p=0.03)$ in ECMO patients.

Conclusions: Although there was no significant association between the use of antibiotics and mortality after overall cardiogenic OHCA treated with TTM, antibiotics may be beneficial in patients who require ECMO.

Keyword: Antibiotics, Cardiac arrest, Extracorporeal membrane oxygenation, Infection, Targeted temperature management

\footnotetext{
* Correspondence: t-tagami@nms.ac.jp

${ }^{1}$ Department of Clinical Epidemiology and Health Economics, School of

Public Health, Graduate School of Medicine, The University of Tokyo, 7-3-1

Hongo, Bunkyo-ku, Tokyo 1138555, Japan

${ }^{2}$ Department of Emergency and Critical Care Medicine, Nippon Medical

School Tama Nagayama Hospital, 1-7-1 Nagayama, Tama-shi, Tokyo 2068512,

Japan

Full list of author information is available at the end of the article
} 


\section{Background}

Patients with return of spontaneous circulation (ROSC) after out-of-hospital cardiac arrest (OHCA) suffer from prolonged, complete, whole-body ischemia and reperfusion, and have a grim prognosis $[1,2]$. Post-cardiac arrest ischemia-reperfusion injury to the brain may reportedly be attenuated by induced hypothermia [3-5]. Thus, the 2015 international guidelines for cardiac arrest recommend a targeted temperature-management (TTM) strategy for adults sustaining an OHCA with an initial shockable rhythm who remain unresponsive after the ROSC [6, 7].

Patients resuscitated after cardiac arrest are reportedly at high risk of developing infectious diseases, especially pneumonia, several days after ROSC [2, 8-13]. Although recent guidelines recommend a TTM strategy $[6,7]$, hypothermia can impair the immune system and increase the incidence of infection $[14,15]$. In addition, invasive medical techniques, such as extracorporeal membrane oxygenation (ECMO), intra-aortic balloon pumping (IABP) and continuous renal replacement therapy (CRRT), are used during TTM and organ support after OHCA [16]. These invasive devices (especially ECMO) may themselves increase the risk of infection [17-19]. Consequently, patients undergoing TTM after cardiac arrest are likely to be at greater risk of infection and death.

Although antibiotic administration is the mainstream treatment for infectious diseases, only a few retrospective studies have suggested a beneficial influence on the survival of OHCA patients who received early antibiotic administration while undergoing TTM [20, 21]. Moreover, there are no current reports of a relationship between early antibiotic administration and mortality among patients with OHCA requiring invasive lifesupporting interventions.

We hypothesized that prophylactic systemic antibiotic therapy during TTM may reduce mortality in OHCA patients, especially in those requiring invasive lifesupporting interventions. The purpose of this study was to evaluate our hypothesis using a large nationwide inpatient database in Japan.

\section{Methods}

Conduct of the study was approved by the Institutional Review Board of The University of Tokyo. Requirement for informed patient consent was waived because of the anonymous nature of the data.

\section{Data source and variables}

We retrospectively evaluated the Japanese Diagnosis Procedure Combination (DPC) database, which was described previously $[16,22,23]$. Briefly, the DPC database includes administrative claims and discharge abstract data for all inpatients discharged from more than 1000 participating hospitals, covering all 82 academic hospitals and more than $90 \%$ of all tertiary-care emergency hospitals in Japan [16, 22, 23]. The database includes the following information for each patient recorded using a uniform data-submission form: age; sex; primary diagnosis, comorbidities on admission, and post-admission complications coded using the International Classification of Diseases, 10th Revision (ICD-10) codes; medical procedures; daily records of all drugs administered and devices used; and discharge status. Patient follow-up began on the day of admission and ended on the date of discharge, either to home, to another hospital, or because of death. Patients with cardiac arrest upon arrival at the hospital were defined as experiencing OHCA, with chest compressions performed on/after arrival. We assumed that cardiac arrest was of cardiac aetiology unless there was an obvious non-cardiac cause (i.e. cerebrovascular disease, respiratory disease, severe trauma, drowning, asphyxiation or drug overdose) [16], in accordance with the International Liaison Committee on Resuscitation consensus statement [24, 25]. We defined patients with non-cardiogenic cardiopulmonary arrest as follows: ICD-10 codes at primary diagnosis and comorbidities upon admission of J60 to J61.9 [subarachnoid and cerebral haemorrhage], I71.0 to I71.9 [aorta dissection and aneurysm], I26.0 to I26.9 [pulmonary embolism], J45.9 and J46 [severe asthma], S00 to T98 [trauma, burns, hanging, accidental hypothermia, drowning, electrocution, anaphylaxis, drug overdose], J69 and T71 [asphyxiation], or K25.0, K25.2, K26.0, K26.2, K27.0, K27.2, K28.0, K28.2, K92.0 to 92.2, and I85.0 to I85.9 [acute gastrointestinal bleeding or oesophageal/gastric varices]).

We designated hospital volume as the number of eligible patients treated for the current study and categorized hospitals into tertiles (i.e. low, medium, and high). The hospital type was categorized as academic or nonacademic. The Japanese Ministry of Health, Labour and Welfare officially approved TTM for patients with cardiac arrest only (i.e. not in other disorders such as traumatic brain injury) in April 2006.

\section{Patient selection and end point}

Adults with cardiogenic OHCA were identified in the DPC database from July 2007 to March 2013. We did not include in-hospital cardiac arrest cases in this study. The inclusion criteria were as follows: (i) age $\geq 18$ years; (ii) diagnosis of OHCA (ICD-10 codes at primary diagnosis and comorbidities on admission of I46.0 [cardiac arrest with successful resuscitation], I46.1 [sudden cardiac death], or I46.9 [cardiac arrest, unspecified]); (iii) and administration of therapeutic hypothermia on days zero and one. Exclusion criteria were as follows: (i) patients with non-cardiogenic cardiopulmonary arrest; (ii) 
patients with malignancy, caducity, and pneumonia upon admission (to maintain comparability between the groups; C00 to C97 [malignancy], R54 [caducity], $\mathrm{J} 13$ to J18, and J690 [bacterial pneumonia and aspiration pneumonia]); (iii) patients discharged within 2 days of admission (to avoid immortal time bias [26]). Thus, we compared patients who received antibiotics within 2 days of admission (antibiotics group) and those who did not receive any antibiotics within that time (control group) [23].

The main endpoint used in this study was all-cause, 30-day in-hospital mortality [25]. We performed subgroup analyses comparing mortality between other groups. We also evaluated the incidence of pneumonia emergence after admission between the two groups.

\section{Statistical analyses}

We performed propensity scoring and instrumental variable analyses. To estimate the propensity score, we fit a logistic regression model designed for antibiotic treatment as a function of background-patient characteristics and in-hospital treatments or interventions performed on day 0 . We included the following: age; sex; hospital type; hospital volume; fiscal year; cardiac arrest on hospital arrival; administration of epinephrine; ventricular fibrillation; defibrillation performed; requirement for PCI, ECMO, IABP and/or CRRT; requirement for catecholamines or vasopressin; and requirement for antiarrhythmic drugs, sivelestat sodium use, and blood transfusion. We performed a one-to-one matched analysis using nearest-neighbour matching based on the estimated propensity scores of the patients. A match occurred when a patient in the antibiotics group had an estimated score within 0.2 standard deviations of a patient in the control group [27]. We examined the balance in baseline variables using standardized differences, where $>10 \%$ was regarded as imbalanced [27]. Descriptive statistics are presented for all patients and propensity score-matched groups. Continuous variables were compared using a t-test. Categorical variables were compared using the chi-square test or Fisher's exact test. We performed logistic regression analysis fitted with generalized estimating equations to examine the association between antibiotics use and survival and accounted for the paired nature of the propensity score-matched patients. We used Cox regression analysis to assess differences in in-hospital survival rates between patients with and without antibiotic treatment in the propensity scorematched groups. Odds ratios (ORs) and hazard ratios (HRs) with $95 \%$ confidence intervals (CIs) were calculated.

We also performed instrumental variable analysis as confirmatory analysis for the propensity score analyses [28]. We used the hospitals' antibiotics prescribing preference as an instrumental variable, and computed the differences in the risk of 30-day mortality between the groups with and those without early antibiotic administration. This approach was implemented using a 2stage least-squares method that also adjusted for patient demographic characteristics. We classified hospitals that administered early antibiotics to the 50th percentile or more of eligible patients as hospitals with a preference for early antibiotics administration, and those that administered to less than the 50th percentile of eligible patients as hospitals without a preference for early antibiotics administration. We estimated the risk difference with its $95 \%$ CI. To confirm that the percentage of hospital use of antibiotics was not a weak instrument, we used a partial $\mathrm{F}$ test. The null hypothesis was that there was no association between patterns of hospital antibiotics use and actual antibiotics use. An F statistic $>10$ suggests that the instrument is not weak [28].

We also performed subgroup analyses to identify the patients who may have benefited the most from antibiotics therapy. We selected relevant subgroups among the propensity score-matched patients and estimated their risk ratios and $95 \%$ CIs for 30-day mortality. For robustness, we further evaluated the subgroup of patients who had the most benefit from early antibiotics administration (i.e. the lowest risk ratio group of patients for 30-day mortality), and performed the same analyses as all patients, including Cox regression and instrumental variable analyses.

A $P$ value $<0.05$ was considered statistically significant. All statistical analyses were performed using IBM SPSS version 22 (IBM Corp., Armonk, NY, USA) and Stata/SE 13.0 (Stata Corp., College Station, TX, USA).

\section{Results}

During the study period, 95,960 OHCA patients were identified in the database. Of the patients initially identified, 2803 eligible cardiogenic patients with OHCA were treated with TTM in 371 hospitals (Fig. 1). These patients were categorized into the antibiotic-treated $(n=$ $1272)$ and control groups $(n=1531)$, and 802 propensity score-matched pairs were generated (Fig. 2). Table 1 shows the demographic and clinical characteristics of the patients in the two groups. In the antibiotics group $(n=1272)$, the most common antibiotics administered were as follows: first-generation cephalosporin for $58.6 \%$ (746 patients), third-generation cephalosporin for $10.4 \%$ (145 patients), and broad-spectrum penicillin for $6.3 \%$ (80 patients).

\section{Overall analysis}

The overall 30-day mortality was $32.5 \%$ (910 of 2803 patients), and the incidence of pneumonia after admission was $14.1 \%$ (395 of 2803 patients). Among unmatched 


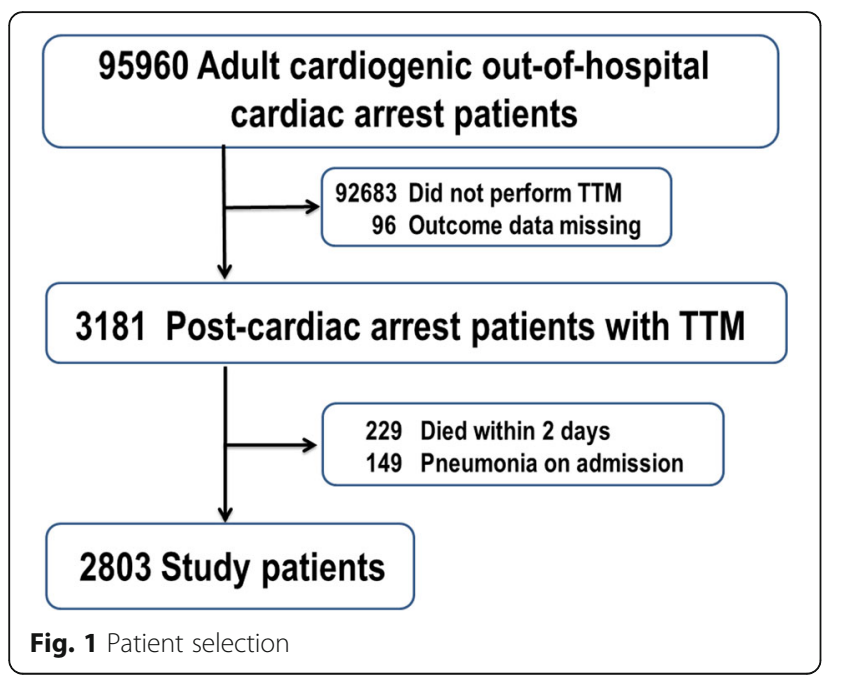

patients, significant differences in 30-day mortality were observed between the two groups $(30.3 \%$ in the control group compared with $35.1 \%$ in the antibiotics-treated group, difference $4.8 \%$; CI $95 \%, 1.2$ to $8.2 \%, p=0.007$ ), but not among matched patients $(33.0 \%$ versus $29.9 \%$, respectively, difference $3.1 \%$; CI $95 \%,-1.4$ to $7.7 \%, p=$ $0.18)$. Logistic regression analyses showed that there was no significant association between the use of antibiotics and lower 30-day mortality in the propensity scorematched groups (OR 0.89; CI $95 \%, 0.73$ to $1.1 ; p=0.24$ ). The Cox regression analysis did not indicate significant differences in-hospital mortality between the control and antibiotics groups for the propensity-matched groups (HR 0.88 , CI $95 \% 0.75$ to $1.04, p=0.12$; Fig. 3 ).

In the instrumental variable model, the null hypothesis of 'no association between patterns of hospital antibiotics use and actual antibiotics use' was rejected $(P<0.001)$. Our instrument of pattern of hospital antibiotics use was not weak (F statistic $=702)$. The estimated reduction in 30-day mortality was not significantly associated with

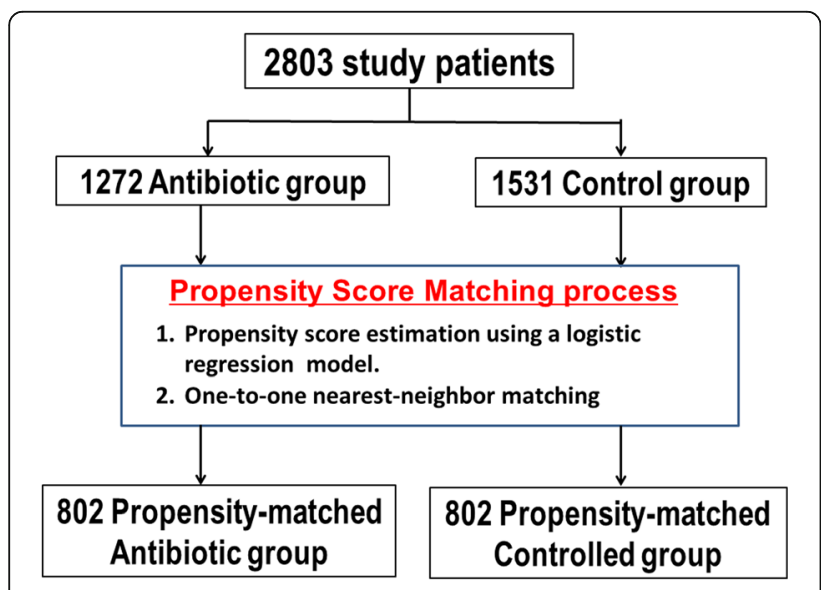

Fig. 2 Propensity score matching process administration of antibiotics $(6.6 \%$, CI $95 \%,-0.5$ to $13.7 \%, p=0.28)$.

Among unmatched patients, there was a significantly lower incidence of pneumonia after admission in the antibiotic group than in the control group (12.6\% vs. $15.3 \%$, difference $-3.7 \%$; CI $95 \%,-0.2$ to $-5.3 \%, p=0.04$ ). However, this difference was not significant in the matched group (13.0 \% vs. $15.8 \%$, difference $-6.3 \%$; CI $95 \%,-0.2$ to $0.6 \%, p=0.10)$.

\section{Subgroup analyses}

The risk of 30-day mortality associated with antibiotics use in the propensity score-matched patients for subgroups is presented in Fig. 4. There was a significant association between the use of antibiotics and lower mortality in the subgroup of patients who required ECMO, IABP, or CRRT. The lowest risk of 30-day mortality associated with the administration of antibiotics was observed in the subgroup who required ECMO (risk ratio 0.67 ; CI $95 \%, 0.50$ to $0.89, p=0.006$ ). Among propensity score-matched patients who did not require ECMO, there was no significant difference in 30-day mortality between the two groups $(28.6 \%$ in the antibiotics group versus $27.8 \%$ in the control group, difference $0.7 \%$; CI $95 \%,-3.9$ to $5.5 \%, p=0.76$ ). In contrast, among patients who required ECMO, there was a significant difference in 30-day mortality between the 2 groups $(62.9 \%$ in the control group versus $43.5 \%$ in the antibiotics group, difference $19.3 \%$; CI $95 \%$, 5.9 to $32.7 \%$; $p=0.005$ ).

Among patients who required ECMO, a logistic regression analysis indicated that there was a significant association between the the early use of antibiotics and lower 30-day mortality in the propensity score-matched groups (OR 0.47, CI $95 \%, 0.27$ to $0.83, p=0.009$ ). Cox regression analysis showed significant mortality differences between the control and antibiotics groups for the propensity-matched groups (HR 0.61, CI $95 \%, 0.43$ to 0.87, $p=0.005$; Fig. 5). In the instrumental variable model, the estimated reduction in 30-day mortality associated with receipt of antibiotics was $18.2 \%$ (CI $95 \%$, 2.1 to $34.4 \%, p=0.03$ ). The lower incidence of pneumonia after admission in the early antibiotics-treated group than in the control group was not significant among patients who required ECMO (9.3\% vs. $13.3 \%$, difference $-4.1 \%$; CI $95 \%,-12.6$ to $4.4 \%, p=0.35$ ).

\section{Discussion}

The results of this study using a nationwide database suggest that there was no significant association between the use of early antibiotics administration and survival in a cohort of patients with OHCA receiving TTM. However, we found a significant association between the use 
Table 1 Patient characteristics, and initial treatments and interventions

\begin{tabular}{|c|c|c|c|c|c|c|c|c|c|c|}
\hline \multirow{3}{*}{$\begin{array}{l}\text { Variable } \\
\text { Age, mean (SD) }\end{array}$} & \multicolumn{5}{|c|}{ Unmatched groups } & \multicolumn{5}{|c|}{ Propensity score-matched groups } \\
\hline & \multicolumn{2}{|c|}{$\begin{array}{l}\text { Control } \\
(n=1531)\end{array}$} & \multicolumn{2}{|c|}{$\begin{array}{l}\text { Antibiotics } \\
(n=1272)\end{array}$} & \multirow{2}{*}{$\begin{array}{l}\text { Standardized } \\
\text { differences, \% } \\
-3.5\end{array}$} & \multicolumn{2}{|c|}{$\begin{array}{l}\text { Control } \\
(n=802)\end{array}$} & \multicolumn{2}{|c|}{$\begin{array}{l}\text { Antibiotics } \\
(n=802)\end{array}$} & \multirow{2}{*}{$\begin{array}{l}\text { Standardized } \\
\text { differences, \% } \\
1.0\end{array}$} \\
\hline & 60.1 & $(15.3)$ & 60.7 & $(14.3)$ & & 60.7 & $(14.8)$ & 60.6 & $(14.6)$ & \\
\hline Sex $(\text { male })^{a}$ & 1,151 & $(75.2)$ & 984 & $(77.4)$ & -5.1 & 613 & $(76.4)$ & 609 & $(75.9)$ & 1.2 \\
\hline Academic hospital & 620 & $(40.5)$ & 392 & $(30.8)$ & 20.3 & 262 & $(32.7)$ & 279 & $(34.8)$ & -4.5 \\
\hline \multicolumn{11}{|l|}{ Hospital volume, cases } \\
\hline Low, $<10$ & 484 & (31.6) & 505 & $(39.7)$ & -16.9 & 317 & (39.5) & 305 & $(38.0)$ & 3.1 \\
\hline Medium 11-22 & 470 & $(30.7)$ & 419 & $(32.9)$ & -4.8 & 271 & $(33.8)$ & 264 & $(32.9)$ & 1.9 \\
\hline High, $>23$ & 577 & $(37.7)$ & 348 & $(27.4)$ & 22.2 & 214 & (26.7) & 233 & $(29.1)$ & -5.3 \\
\hline Ventricular fibrillation & 746 & $(48.7)$ & 645 & $(50.7)$ & -4.0 & 397 & $(49.5)$ & 413 & $(51.5)$ & -4.0 \\
\hline Required defibrillation on admission & 328 & $(21.4)$ & 409 & $(32.2)$ & -24.4 & 186 & $(23.2)$ & 204 & $(25.4)$ & -5.2 \\
\hline Cardiac arrest on admission & 540 & $(35.3)$ & 586 & $(46.1)$ & -22.1 & 320 & (39.9) & 319 & $(39.8)$ & 0.3 \\
\hline Epinephrine provided on admission & 597 & $(39.0)$ & 617 & $(48.5)$ & -19.3 & 342 & (42.6) & 338 & $(42.1)$ & 1.0 \\
\hline Percutaneous coronary intervention & 872 & $(57.0)$ & 902 & $(70.9)$ & -29.4 & 507 & $(63.2)$ & 536 & $(66.8)$ & -7.6 \\
\hline Intra-aortic balloon pumping & 308 & $(20.1)$ & 483 & $(38.0)$ & -40.1 & 218 & $(27.2)$ & 204 & $(25.4)$ & 4.0 \\
\hline Continuous renal replacement therapy & 136 & $(8.9)$ & 248 & $(19.5)$ & -30.8 & 99 & $(12.3)$ & 104 & $(13.0)$ & -1.9 \\
\hline $\begin{array}{l}\text { Extracorporeal membrane oxygenation system } \\
\text { Pharmacologic intervention }\end{array}$ & 147 & (9.6) & 298 & $(23.4)$ & -37.9 & 105 & $(13.1)$ & 108 & $(13.5)$ & -1.1 \\
\hline Dopamine & 741 & $(48.4)$ & 703 & $(55.3)$ & -13.8 & 408 & $(50.9)$ & 425 & $(53.0)$ & -4.2 \\
\hline Dobutamine & 286 & $(18.7)$ & 403 & $(31.7)$ & -30.3 & 189 & (23.6) & 176 & $(21.9)$ & 3.9 \\
\hline Norepinephrine & 487 & (31.8) & 584 & $(45.9)$ & -29.2 & 300 & (37.4) & 300 & $(37.4)$ & 0.0 \\
\hline Vasopressin & 51 & (3.3) & 53 & $(4.2)$ & -4.4 & 28 & (3.5) & 26 & $(3.2)$ & 1.4 \\
\hline Amiodarone & 371 & $(24.2)$ & 463 & $(36.4)$ & -26.7 & 234 & $(29.2)$ & 244 & $(30.4)$ & -2.7 \\
\hline Nifekalant & 76 & $(5.0)$ & 103 & $(8.1)$ & -12.7 & 45 & (5.6) & 57 & $(7.1)$ & -6.1 \\
\hline Lidocaine & 370 & $(24.2)$ & 455 & (35.8) & -25.5 & 233 & (29.1) & 241 & $(30.0)$ & -2.2 \\
\hline Sivelestat sodium & 44 & (2.9) & 84 & $(6.6)$ & -17.6 & 32 & $(4.0)$ & 33 & $(4.1)$ & -0.6 \\
\hline \multicolumn{11}{|l|}{ Blood transfusion } \\
\hline Red blood cells & 133 & $(8.7)$ & 268 & $(21.1)$ & -35.3 & 92 & (11.5) & 99 & $(12.3)$ & -2.7 \\
\hline Fresh frozen plasma & 86 & (5.6) & 199 & $(15.6)$ & -33.0 & 59 & $(7.4)$ & 66 & $(8.2)$ & -3.3 \\
\hline Platelets & 21 & (1.4) & 61 & $(4.8)$ & -19.9 & 16 & $(2.0)$ & 15 & (1.9) & 0.9 \\
\hline
\end{tabular}

anumbers in parentheses are proportions (\%) unless otherwise stated

of antibiotics and lower mortality in the subgroup of patients who required ECMO, IABP, or CRRT.

We analysed real-world clinical data of more than 2,800 adults with cardiogenic OHCA treated at 371 hospitals. In the present study, the decision to provide antibiotics was left to the discretion of physician of each hospital. To date, there have been no randomised trials or nation-wide studies regarding this issue. Accordingly, there are no guidelines whether to provide antibiotics for patients after cardiac arrest due to the lack of data. Approximately, $45 \%$ of the patients received antibiotics in the present study. Although analysis of the baseline patient characteristics in the unmatched group showed that antibiotics were used in a greater proportion of patients with more severe status after cardiac arrest (i.e. those at greater risk of death and more likely to require defibrillation upon admission, PCI, IABP, ECMO, catecholamines, antiarrhythmic medications, or blood products), one-to-one propensity score-matching successfully balanced the characteristics of the antibiotic and control groups. Although patients receiving antibiotics appeared less likely to die than did similar patients who did not receive antibiotics (mortality rates 33.0 and $29.9 \%$, respectively), this difference was not statistically significant.

Similar results were observed in the instrumental variable analysis, which also found that there was no significant reduction in 30-day mortality associated with the receipt of antibiotics. When hospitals have highly consistent antibiotic-use policies for post-cardiac arrest patients treated with a TTM strategy, decisions regarding antibiotic use may be made independently of individual patient characteristics, including unmeasured variables, 


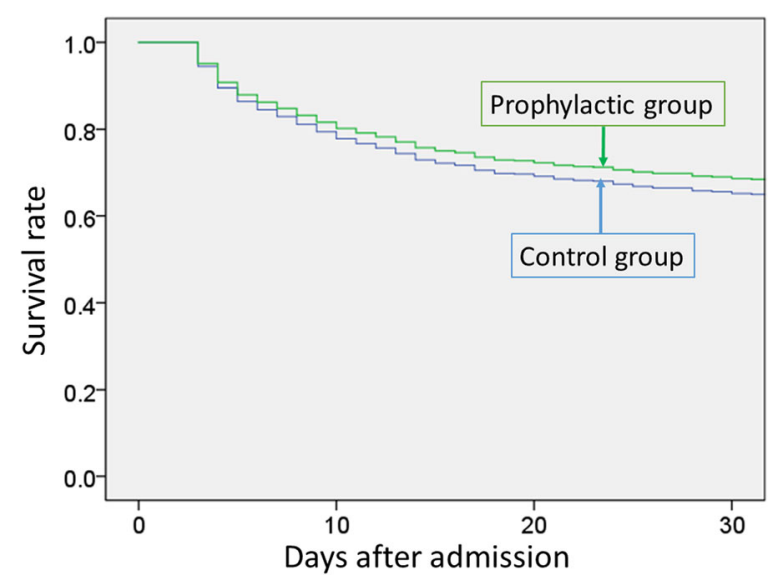

Fig. 3 Survival plots for all patients treated with or without early antibiotics administration in propensity score-matched groups.

There was no significant difference in 30-day mortality between the antibiotics and control groups. (Cox regression analysis; hazard ratio, 0.88 ; confidence interval $95 \%, 0.75$ to 1.04 )

as OHCA occurs independent of the location of the admitting hospital. In this situation, the hospital's antibiotics use policy may act as an instrumental variable, thereby setting the conditions for a 'natural experiment' that allows for an unbiased estimate of risk in patients with OHCA undergoing TTM. Considering the results of the propensity score and instrumental variable analyses, early antibiotic-administration appears to have little influence on survival during TTM after OHCA. Our negative results of the present study may have resulted from a lack of statistical power. However, the absolute difference in 30-day mortality in the propensity-matched analysis was $3.1 \%$, thereby suggesting that at least 7272 patients (3636 patients per group) would be required to show a significant difference in future trials $(\alpha$ error $=$ $0.05,1-\beta$ [power] $=0.8$ ).

The use of ECMO in the intensive care unit has substantially increased over the past $\sim 10$ years $[17,29,30]$, including during cardiopulmonary resuscitation (CPR) and afterwards [16, 31, 32]. The results of a recent metaanalysis by Xie et al. [33] suggested that ECMO has the potential to improve outcomes after cardiac arrest; however, they also suggested that ECMO is associated with significant complication rates, including infection, which must be incorporated into the risk-benefit analysis when considering treatment [33]. The latest resuscitation guidelines state that extracorporeal CPR is a reasonable rescue therapy for selected patients with cardiac arrest when initial conventional CPR is failing; however, they provide no guidance regarding the role of antibiotic administration [6, 7].

A low cardiac output state combined with high systemic vascular resistance results in poor systemic perfusion in OHCA patients who successfully achieve ROSC.
Post-cardiac arrest acute kidney injury occurs in more than $50 \%$ of cardiac arrest patients [3]. Potential treatments to counteract this severe hemodynamic status and acute renal failure include IABP and CRRT. Although these devices themselves may increase the risk of infection, the use of prophylactic antibiotics is controversial [18]. No studies have evaluated the relationship between early antibiotic administration and mortality among OHCA patients requiring these invasive life-supporting interventions.

We found that patients with cardiogenic OHCA who required ECMO, IABP, or CRRT might benefit from early antibiotic treatment in our propensity score and instrumental variable analyses. Early antibiotic administration may have the greatest therapeutic benefits in patients with the most severe OHCA patients with TTM. Grimaldi et al. [34] recently reported that nearly half of the patients with post-cardiac arrest shock had high blood endotoxin concentration in the first $12 \mathrm{~h}$ following ROSC. They also reported that endotoxemia was associated with shock duration, mean daily dose of vasopressors and organ failure [34]. Thus, among those patients with OHCA and severe hemodynamic disturbance to require ECMO, IABP, and/ or CRRT, early antibiotics administration may have the potential to improve outcomes.

Another explanation could be that ECMO, IABP, and/ or CRRT themselves increase the risk of nosocomial infection. Patients on ECMO for extracorporeal CPR reportedly developed 24.7 infections per 1000 ECMO days according to data from the Extracorporeal Life Support Organization Registry [35], and these infections were associated with increased mortality. Nevertheless, the Task Force does not currently recommend routine antibiotic administration during ECMO, due to lack of evidence of its efficacy [17]. Furthermore, previous studies showed that the incidence of catheter-related bacteremia in patients who required hemodialysis ranged between 0.6 and 6.5 episodes per 1000 catheter days, and increased linearly with the duration of catheter use $[18,36]$. Although we cannot infer a robust cause-and-effect relationship from this study, we speculate that there may be an association between early antibiotic administration and improved survival in OHCA patients requiring ECMO, IABP, and/or CRRT during TTM. We believe that our nationwide retrospective data will be useful when planning for future international trials.

Although the incidence of pneumonia after OHCA is inconsistent across previous studies, the incidence of pneumonia in the present report may be lower than that of previous reports outside Japan $[8,10,15,20,21]$. This may be due to several reasons. Firstly, because of its retrospective design, the diagnosis of pneumonia was assigned to the doctors in charge of each 371 hospitals. Thus, there might be some under-reporting (or over- 


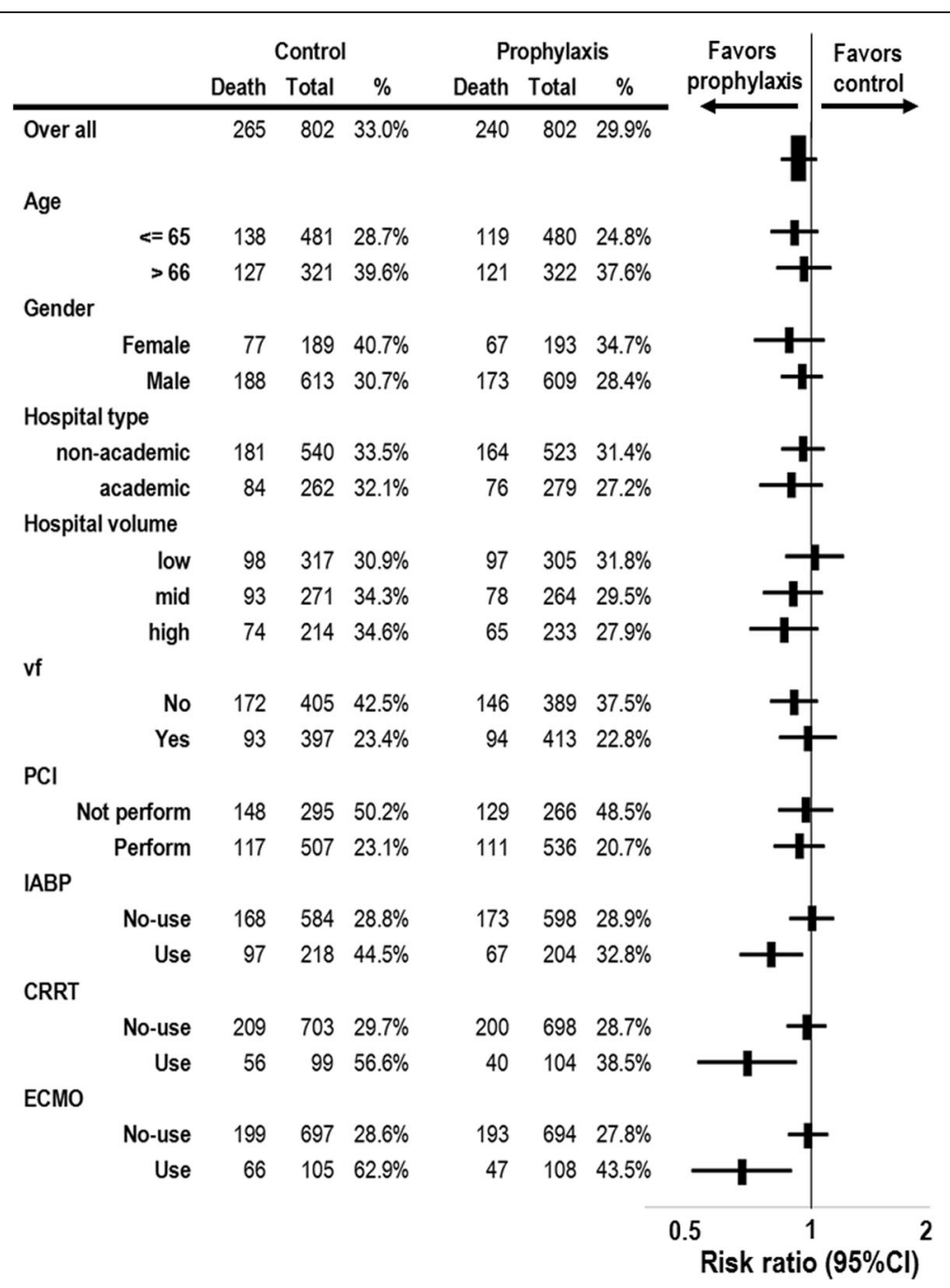

Fig. 4 Risk ratios of in-hospital mortality associated with antibiotic use in propensity score-matched patients. CRRT, continuous renal replacement therapy; ECMO, extracorporeal membrane oxygenation; IABP, intra-aortic balloon pumping; PCI, percutaneous coronary angiography/intervention; vf, ventricular fibrillation

reporting) in the current results. Secondly, in the present study, we included only cardiogenic OHCA patients that underwent TTM. In Japan, during the study period, most TTMs were managed with a milder target temperature for a longer period (e.g. duration of cooling for $>2$ days in $34^{\circ}$ $\mathrm{C}$ and $>2$ days for rewarming $[32,37])$, than the recommendations in international guidelines published during the study period (i.e. cooling to $32{ }^{\circ} \mathrm{C}$ to $34{ }^{\circ} \mathrm{C}$ for 12 or $24 \mathrm{~h}$ [38]). Due to these differences, both the incidence and recognition of pneumonia by the attending doctors may have decreased. Further studies are required to clarify our speculations.

Our study has several limitations. Firstly, although we evaluated nationwide data using a propensity score and instrumental variable analyses, the study was conducted retrospectively. Therefore, a cause-and-effect relationship cannot be established. Large randomized trials are necessary to confirm our findings; however, such studies are challenging in this vulnerable population. Indeed, the mortality rate exceeds $50 \%$ in those who underwent ECMO. Secondly, a limited number of covariates could be analysed as the database did not include several important factors, particularly pre-hospital data including whether the OHCA was witnessed by a bystander, the quality of resuscitation given, whether resuscitation was dispatcher-assisted, and how the emergency services treated the patients [39]. Although we endeavoured to compensate for these potential unmeasured confounders using instrumental variable analyses, further studies are required. Thirdly, we evaluated cardiogenic OHCA patients who underwent therapeutic hypothermia without pneumonia on hospital arrival in the present study. However, since pneumonia often presents gradually after admission, there might be some undiagnosed or underreported 


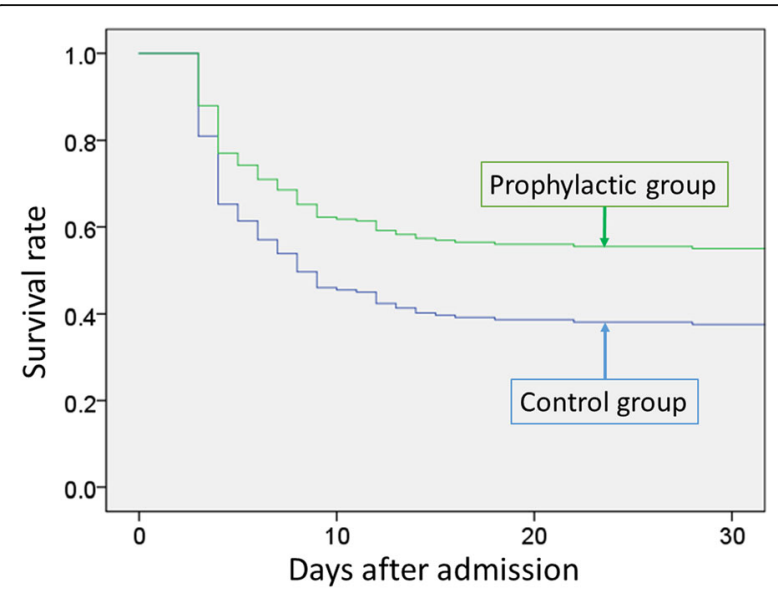

Fig. 5 Survival plots for a subgroup of patients who required extracorporeal membrane oxygenation in the propensity scorematched groups. There was a significant difference in 30-day mortality between the antibiotics and control groups (Cox regression analysis; hazard ratio, $0.61 ; 95 \%$ confidence interval, 0.43 to 0.87 )

pneumonia. In addition, we could not identify catheterrelated infections from the current database. Fourthly, the DPC is an administrative database with information input in relation to patient discharge. We could not follow up patients after discharge from the hospital because this information was not available in the database. Finally, we evaluated only adults with cardiogenic OHCA treated by TTM. Our results cannot be generalized to the use of prophylactic or early administration of antibiotics for children, adults with non-cardiogenic OHCA (especially who already had pneumonia on admission), in-hospital cardiac arrest patients, or those not treated with TTM.

\section{Conclusions}

Although there was no significant association between the use of early antibiotics administration and mortality in a large cohort of patients with cardiogenic OHCA undergoing TTM, antibiotics appear to improve survival in a subgroup of patients that require ECMO. Further studies are required to confirm our results.

\section{Abbreviations \\ CPR: Cardiopulmonary resuscitation; CRRT: Continuous renal replacement therapy; ECMO: Extracorporeal membrane oxygenation; IABP: Intra-aortic balloon pumping; OHCA: Out-of-hospital cardiac arrest; PCI: Percutaneous coronary angiography/intervention; TTM: Targeted temperature management; vf: ventricular fibrillation;}

\section{Acknowledgements}

This work was supported by Grants for Research on Policy Planning and Evaluation from the Ministry of Health, Labour and Welfare, Japan (Grant Numbers: H28-Policy-Designated-009 to Prof. Fushimi and Prof. Yasunaga; H27-Policy-Strategy-011 to Prof. Yasunaga) and a Grant-in-Aid for Scientific Research, Japan (Grant Number: KAKENHI-15H05685 to Dr. Tagami). The funders had no role in the execution of this study or interpretation of the results.

\section{Availability of data and materials}

We used the data from Japanese Diagnosis Procedure Combination (DPC) database, the data cannot be shared due to restriction of our internal rule.

\section{Authors' contributions}

$\Pi \pi$ contributed to the design and conception of the study; acquisition, analysis and interpretation of data; and manuscript preparation. HM contributed to design and conception of the study; acquisition, analysis and interpretation of data; and critical review of the manuscript. MK contributed to design and conception of the study, and critical review of the manuscript. YM contributed to design and conception of the study, and critical review of the manuscript. JK contributed to design and conception of the study, and critical review of the manuscript. Yl contributed to design and conception of the study, and critical review of the manuscript. KU contributed to design and conception of the study, and critical review of the manuscript. KF contributed to design and conception of the study, acquisition of data, and critical review of the manuscript. HY contributed to the design and conception of the study; acquisition, analysis and interpretation of data; and manuscript preparation. All authors read and approved the final manuscript.

\section{Competing interests}

The authors declare that they have no competing interests.

\section{Consent for publication}

Not applicable.

\section{Ethics approval and consent to participate}

Conduct of the study was approved by the Institutional Review Board of The University of Tokyo. Requirement for informed patient consent was waived because of the anonymous nature of the data.

\section{Author details}

'Department of Clinical Epidemiology and Health Economics, School of Public Health, Graduate School of Medicine, The University of Tokyo, 7-3-1 Hongo, Bunkyo-ku, Tokyo 1138555, Japan. ${ }^{2}$ Department of Emergency and Critical Care Medicine, Nippon Medical School Tama Nagayama Hospital, 1-7-1 Nagayama, Tama-shi, Tokyo 2068512, Japan. ${ }^{3}$ Department of Health Informatics and Policy, Graduate School of Medicine, Tokyo Medical and Dental University, 1-5-45 Yushima, Bunkyoku, Tokyo 1138510, Japan.

Received: 2 June 2016 Accepted: 28 September 2016

Published online: 07 October 2016

\section{References}

1. Kudenchuk PJ, Sandroni C, Drinhaus HR, Bottiger BW, Cariou A, Sunde K, et al. Breakthrough in cardiac arrest: reports from the 4th Paris International Conference. Ann Intensive Care. 2015;5(1):22.

2. Neumar RW, Nolan JP, Adrie C, Aibiki M, Berg RA, Bottiger BW, et al. Postcardiac arrest syndrome: epidemiology, pathophysiology, treatment, and prognostication. A consensus statement from the International Liaison Committee on Resuscitation (American Heart Association, Australian and New Zealand Council on Resuscitation, European Resuscitation Council, Heart and Stroke Foundation of Canada, InterAmerican Heart Foundation, Resuscitation Council of Asia, and the Resuscitation Council of Southern Africa); the American Heart Association Emergency Cardiovascular Care Committee; the Council on Cardiovascular Surgery and Anesthesia; the Council on Cardiopulmonary, Perioperative, and Critical Care; the Council on Clinical Cardiology; and the Stroke Council. Circulation. 2008;118(23):2452-83.

3. Bernard SA, Gray TW, Buist MD, Jones BM, Silvester W, Gutteridge G, et al. Treatment of comatose survivors of out-of-hospital cardiac arrest with induced hypothermia. N Engl J Med. 2002;346(8):557-63.

4. Hypothermia after Cardiac Arrest Study G. Mild therapeutic hypothermia to improve the neurologic outcome after cardiac arrest. N Engl J Med. 2002;346(8):549-56.

5. Perman SM, Goyal M, Neumar RW, Topjian AA, Gaieski DF. Clinical applications of targeted temperature management. Chest. 2014;145(2): 386-93.

6. Callaway CW, Soar J, Aibiki M, Bottiger BW, Brooks SC, Deakin CD, et al. Part 4: Advanced Life Support: 2015 International consensus on cardiopulmonary resuscitation and emergency cardiovascular care science with treatment recommendations. Circulation. 2015;132(16 Suppl 1):S84-145. 
7. Soar J, Nolan JP, Bottiger BW, Perkins GD, Lott C, Carli P, et al. European resuscitation council guidelines for resuscitation 2015: Section 3. Adult advanced life support. Resuscitation. 2015;95:100-47.

8. Perbet S, Mongardon N, Dumas F, Bruel C, Lemiale V, Mourvillier B, et al. Early-onset pneumonia after cardiac arrest: characteristics, risk factors and influence on prognosis. Am J Respir Crit Care Med. 2011;184(9):1048-54.

9. Woo JH, Lim YS, Yang HJ, Park WB, Cho JS, Kim JJ, et al. Factors associated with pneumonia in post-cardiac arrest patients receiving therapeutic hypothermia. Am J Emerg Med. 2014;32(2):150-5.

10. Kakavas S, Mongardon N, Cariou A, Gulati A, Xanthos T. Early-onset pneumonia after out-of-hospital cardiac arrest. J Infect. 2015;70(6):553-62.

11. Tsai MS, Chiang WC, Lee CC, Hsieh CC, Ko PC, Hsu CY, et al. Infections in the survivors of out-of-hospital cardiac arrest in the first 7 days. Intensive Care Med. 2005;31(5):621-6.

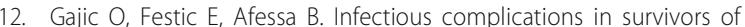
cardiac arrest admitted to the medical intensive care unit. Resuscitation. 2004;60(1):65-9.

13. Mongardon N, Dumas F, Ricome S, Grimaldi D, Hissem T, Pene F, et al. Postcardiac arrest syndrome: from immediate resuscitation to long-term outcome. Ann Intensive Care. 2011;1(1):45.

14. Geurts M, Macleod MR, Kollmar R, Kremer PH, van der Worp HB. Therapeutic hypothermia and the risk of infection: a systematic review and metaanalysis. Crit Care Med. 2014;42(2):231-42

15. Mongardon N, Perbet S, Lemiale V, Dumas F, Poupet $H$, Charpentier J, et al. Infectious complications in out-of-hospital cardiac arrest patients in the therapeutic hypothermia era. Crit Care Med. 2011;39(6):1359-64.

16. Tagami T, Matsui H, Fushimi K, Yasunaga H. Changes in therapeutic hypothermia and coronary intervention provision and in-hospital mortality of patients with out-of-hospital cardiac arrest: a Nationwide Database Study. Crit Care Med. 2016:44(3):488-95.

17. O'Horo JC, Cawcutt KA, De Moraes AG, Sampathkumar P, Schears GJ. The evidence base for prophylactic antibiotics in patients receiving extracorporeal membrane oxygenation. ASAIO J. 2016;62(1):6-10.

18. Silva TN, de Marchi D, Mendes ML, Barretti P, Ponce D. Approach to prophylactic measures for central venous catheter-related infections in hemodialysis: a critical review. Hemodial Int. 2014;18(1):15-23.

19. Cheng R, Hachamovitch R, Kittleson M, Patel J, Arabia F, Moriguchi J, et al. Complications of extracorporeal membrane oxygenation for treatment of cardiogenic shock and cardiac arrest: a meta-analysis of 1,866 adult patients. Ann Thorac Surg. 2014:97(2):610-6.

20. Gagnon DJ, Nielsen N, Fraser GL, Riker RR, Dziodzio J, Sunde K, et al. Prophylactic antibiotics are associated with a lower incidence of pneumonia in cardiac arrest survivors treated with targeted temperature management. Resuscitation. 2015;92:154-9.

21. Hellenkamp K, Onimischewski S, Kruppa J, Fasshauer M, Becker A, Eiffert H, et al. Early pneumonia and timing of antibiotic therapy in patients after nontraumatic out-of-hospital cardiac arrest. Crit Care. 2016;20(1):31.

22. Tagami T, Matsui H, Fushimi K, Yasunaga H. Low-dose corticosteroid treatment and mortality in refractory abdominal septic shock after emergency laparotomy. Ann Intensive Care. 2015;5(1):32.

23. Tagami T, Matsui $H$, Fushimi $\mathrm{K}$, Yasunaga $\mathrm{H}$. Prophylactic antibiotics may improve outcome in patients with severe burns requiring mechanical ventilation: propensity score analysis of a Japanese Nationwide database. Clin Infect Dis. 2016;62(1):60-6.

24. Jacobs I, Nadkarni V, Bahr J, Berg RA, Billi JE, Bossaert L, et al. Cardiac arrest and cardiopulmonary resuscitation outcome reports: update and simplification of the Utstein templates for resuscitation registries: a statement for healthcare professionals from a task force of the International Liaison Committee on Resuscitation (American Heart Association, European Resuscitation Council, Australian Resuscitation Council, New Zealand Resuscitation Council, Heart and Stroke Foundation of Canada, InterAmerican Heart Foundation, Resuscitation Councils of Southern Africa). Circulation. 2004;110(21):3385-97.

25. Perkins GD, Jacobs IG, Nadkarni VM, Berg RA, Bhanji F, Biarent D, et al. Cardiac arrest and cardiopulmonary resuscitation outcome reports: update of the Utstein Resuscitation Registry Templates for Out-of-Hospital Cardiac Arrest: a statement for healthcare professionals from a task force of the International Liaison Committee on Resuscitation (American Heart Association, European Resuscitation Council, Australian and New Zealand Council on Resuscitation, Heart and Stroke Foundation of Canada, InterAmerican Heart Foundation, Resuscitation Council of Southern Africa,
Resuscitation Council of Asia); and the American Heart Association Emergency Cardiovascular Care Committee and the Council on Cardiopulmonary, Critical Care, Perioperative and Resuscitation. Circulation. 2015;132(13):1286-300

26. Suissa S. Immortal time bias in pharmaco-epidemiology. Am J Epidemiol. 2008;167(4):492-9.

27. Austin PC. The use of propensity score methods with survival or time-toevent outcomes: reporting measures of effect similar to those used in randomized experiments. Stat Med. 2014;33(7):1242-58.

28. Staiger D, Stock JH. Instrumental Variables Regression with Weak Instruments. Econometrica. 1994:65(3):557-86.

29. Sauer CM, Yuh DD, Bonde P. Extracorporeal membrane oxygenation use has increased by 433\% in adults in the United States from 2006 to 2011. ASAIO J. 2015;61(1):31-6.

30. Richard C, Argaud L, Blet A, Boulain T, Contentin L, Dechartres A, et al. Extracorporeal life support for patients with acute respiratory distress syndrome: report of a Consensus Conference. Ann Intensive Care. 2014:4:15.

31. Group S-KS. Changes in treatments and outcomes among elderly patients with out-of-hospital cardiac arrest between 2002 and 2012: A post hoc analysis of the SOS-KANTO 2002 and 2012. Resuscitation. 2015;97:76-82.

32. Sakamoto T, Morimura N, Nagao K, Asai Y, Yokota H, Nara S, et al. Extracorporeal cardiopulmonary resuscitation versus conventional cardiopulmonary resuscitation in adults with out-of-hospital cardiac arrest: a prospective observational study. Resuscitation. 2014:85(6):762-8.

33. Xie A, Phan K, Tsai YC, Yan TD, Forrest P. Venoarterial extracorporeal membrane oxygenation for cardiogenic shock and cardiac arrest: a metaanalysis. J Cardiothorac Vasc Anesth. 2015;29(3):637-45.

34. Grimaldi D, Sauneuf B, Guivarch E, Ricome S, Geri G, Charpentier J, et al. High level of endotoxemia following out-of-hospital cardiac arrest is associated with severity and duration of postcardiac arrest shock. Crit Care Med. 2015;43(12):2597-604.

35. Bizzarro MJ, Conrad SA, Kaufman DA, Rycus P. Extracorporeal life support organization task force on infections EMO. Infections acquired during extracorporeal membrane oxygenation in neonates, children, and adults. Pediatr Crit Care Med. 2011;12(3):277-81.

36. Lok CE, Mokrzycki MH. Prevention and management of catheter-related infection in hemodialysis patients. Kidney Int. 2011;79(6):587-98.

37. Yokoyama H, Nagao K, Hase M, Tahara Y, Hazui H, Arimoto H, et al. Impact of therapeutic hypothermia in the treatment of patients with out-ofhospital cardiac arrest from the J-PULSE-HYPO study registry. Circ J. 2011; 75(5):1063-70.

38. Peberdy MA, Callaway CW, Neumar RW, Geocadin RG, Zimmerman JL, Donnino M, et al. Part 9: post-cardiac arrest care: 2010 American Heart Association Guidelines for Cardiopulmonary Resuscitation and Emergency Cardiovascular Care. Circulation. 2010;122(18 Suppl 3):S768-86.

39. Orban JC, Giolito D, Tosi J, Le Duff F, Boissier N, Mamino C, et al. Factors associated with initiation of medical advanced cardiac life support after outof-hospital cardiac arrest. Ann Intensive Care. 2016:6(1):12.

\section{Submit your next manuscript to BioMed Central and we will help you at every step:}

- We accept pre-submission inquiries

- Our selector tool helps you to find the most relevant journal

- We provide round the clock customer support

- Convenient online submission

- Thorough peer review

- Inclusion in PubMed and all major indexing services

- Maximum visibility for your research

Submit your manuscript at www biomedcentral.com/submit
C BioMed Central 